\title{
Eficacia y seguridad de la combinación de fexofenadina con montelukast en el tratamiento de la rinitis alérgica
}

\author{
Efficacy and safety of combined therapy of fexofenadine and \\ montelukast for the treatment of allergic rhinitis
}

\author{
Patricia O'farrill-Romanillos', Magda Luz Atrián-Salazar², Leonel González-González², \\ Diana Molina-Vélez ${ }^{3}$, Elizabeth Meda-Monzón ${ }^{3}$, Concepción García-Morales ${ }^{4}$, \\ Vanessa Cohen-Muñoz ${ }^{5}$, Gabriela Sánchez-Casado ${ }^{5}$
}

\section{RESUMEN}

El propósito de este trabajo fue revisar la literatura científica que evalúa la eficacia y seguridad de las monoterapias de fexofenadina y montelukast, la terapia combinada (fija o en asociación) de montelukast - fexofenadina, así como de montelukast con otros antihistamínicos de segunda generación en el tratamiento de la rinitis alérgica. Se realizó una estrategia de búsqueda bibliográfica de múltiples etapas, en donde se identificaron estudios basados en ensayos clínicos y estudios no aleatorizados (ensayo controlado no aleatorizado, controlado antes-después, de series de tiempo interrumpidas, con controles históricos, de cohorte, de casos y controles, estudio transversal, y series de casos) en pacientes con rinitis alérgica, en las bases de datos MEDLINE/ PubMed, Scopus, Web of Science, Biblioteca Cochrane, Redalyc y Colección BVS y debido a la cantidad de resultados obtenidos se incluyó la búsqueda en Hinari. Con base en esta revisión se concluye que las combinaciones de antihistamínicos de segunda generación y antagonistas de leucotrienos y, en particular, la combinación fija de fexofenadina - montelukast es eficaz, segura y favorece la adherencia al tratamiento, y a largo plazo también ayuda a alcanzar el objetivo terapéutico.

Palabras clave: Rinitis alérgica estacional, rinitis alérgica perenne, antagonistas de los receptores histamínicos, eficacia, seguridad.

\begin{abstract}
The purpose of this work was to review the scientific literature that evaluates the efficacy and safety of monotherapies of fexofenadine and montelukast, the combined therapy (fixed-dose or separate drug combinations) of montelukast-fexofenadine, as well as the use of montelukast together with other second-generation antihistamines in the treatment of allergic rhinitis. A multistage literature search strategy was designed, including clinical trials and non-randomized studies (non-randomized controlled trial, controlled before-after study, interrupted time series study, historical control study, cohort study, case-control study, crosssectional study, and case series) evaluating patients with allergic rhinitis. The databases MEDLINE/PubMed, Scopus, Web of Science, Cochrane Library, Redalyc, BVS Collection, and, due to the number of results obtained, Hinari were included. Based on this review, the conclusion is that the combinations of secondgeneration antihistamines with leukotriene antagonists and, in particular, the fixed combination of fexofenadine-montelukast are effective, safe and promote treatment adherence. In the long term, they also help achieve therapeutic goals.
\end{abstract}

Keywords: Seasonal allergic rhinitis, perennial allergic rhinitis, histamine antagonists, efficacy, safety.

\footnotetext{
1. Instituto Mexicano del Seguro Social, Servicio de Alergia e Inmunología Clínica, Hospital de Especialidades, Centro Médico Nacional Siglo XXI - Mexico City - CDMX - México.

2. Instituto Nacional de Salud Pública, Dirección de Planeación - Cuernavaca, Morelos, México.

3. Instituto Nacional de Salud Pública, Centro de Investigación en Evaluación y Encuestas - Cuernavaca, Morelos, México.

4. Comisión para la Protección contra los Riesgos Sanitarios del Estado de Morelos, Epidemiología - Cuernavaca, Morelos, México.

5. Sanofi - México, Consumer Health Care - Mexico City, CDMX, México.
}

Financiamiento: El desarrollo y la publicación de esta revisión fueron financiados por Sanofi Aventis de México, S.A. de C.V. 


\section{Introducción}

Entre las enfermedades respiratorias crónicas que se presentan con mayor frecuencia se encuentran las alergias respiratorias; la más común es la rinitis alérgica (RA), ${ }^{1-3}$ una enfermedad inflamatoria de la mucosa nasal, mediada por la inmunoglobulina $E$ ( $\operatorname{lgE})$, como respuesta del sistema inmunitario al inhalar diversos alérgenos. ${ }^{4,5}$

Los principales aeroalérgenos involucrados en la RA son: ácaros del polvo doméstico; epitelio de animales; pólenes, incluidos los pastos, malezas como Parietaria, Ambrosia, Artemisia, árboles, como abedul, olivo, avellana y ciprés; hongos, cucarachas; y como factores irritantes, los contaminantes del aire, como partículas residuales de combustión de diesel.4,6

De acuerdo con la European Academy of Allergy and Clinical Immunology (EAACl) se diagnostica a partir de la presencia de dos o más síntomas nasales por más de 1 hora al día. ${ }^{4}$

El diagnóstico es clínico y se caracteriza por la presencia de los siguientes síntomas: congestión nasal, rinorrea hialina, prurito nasal y ocular, además de estornudos paroxísticos, ${ }^{4}$ la evidencia de sensibilización a través de pruebas in vivo, como las pruebas cutáneas, o in vitro, como la determinación en sangre de IgE específica a aeroalérgenos. Es fundamental la correlación clínica entre la presencia de los síntomas y la exposición al alérgeno. ${ }^{4}$

Se han propuesto diversas clasificaciones para la $R A,{ }^{7,8}$ siendo que una de las primeras se realizó en 1999 con base en el tiempo de exposición, subdividida en: perenne (PAR, por sus siglas en inglés), estacional (SAR) y ocupacional; sin embargo, no era satisfactoria debido a que no se ajustaba a la vida real por diversos motivos relacionados con los alérgenos, por lo que desde 2001 la Allergic Rhinitis and its Impact on Asthma (ARIA) la clasificó de acuerdo a la temporalidad ( \pm 4 días a la semana, \pm 4 semanas) en persistente $o$ intermitente, y a la severidad (interferencia en actividades cotidianas y sueño), en leve o moderada a severa. ${ }^{9}$

El objetivo del tratamiento es la mejoría en los síntomas. Como parte de la primera línea se incluye evitar el contacto con los alérgenos (en los casos en los que es posible), así como a irritantes. Se identifican dos tipos de tratamiento, el farmacológico con uso de antihistamínicos $\mathrm{H} 1$ de segunda generación orales o tópicos, corticoesteroides nasales y antagonistas de leucotrienos, combinaciones de esteroide y antihistamínicos tópicos nasales, así como antihistamínicos con antagonistas de leucotrienos orales ${ }^{9-12}$ (Figura 1) y la inmunoterapia alérgeno específica, de acuerdo al resultado de pruebas cutáneas, que puede ser por vía subcutánea o sublingual, y que consiste en la administración de dosis crecientes del alérgeno con el objetivo de inducir tolerancia al mismo.4,13

El propósito de este trabajo fue revisar la literatura científica que evalúa la eficacia y seguridad de las monoterapias de fexofenadina y montelukast, la terapia combinada (fija o en asociación) de montelukast - fexofenadina, así como de montelukast con otros antihistamínicos de segunda generación en el tratamiento de la rinitis alérgica.

\section{Métodos}

Se realizó una estrategia de búsqueda bibliográfica de múltiples etapas, en donde se identificaron estudios basados en ensayos clínicos y estudios no aleatorizados (ensayo controlado no aleatorizado, estudio controlado antes-después, estudio de series de tiempo interrumpidas, estudio con controles históricos, estudio de cohortes, estudio de casos y controles, estudio transversal, series de casos) sobre RA, en las bases de datos: MEDLINE/PubMed, Scopus, Web of Science, Biblioteca Cochrane, Redalyc y Colección BVS y debido a la cantidad de resultados obtenidos se incluyó la búsqueda en Hinari.

Se empleó el enfoque del vocabulario controlado de términos $\mathrm{MeSH}$; la estrategia para búsqueda en todas las bases de datos fue adaptada de aquella utilizada para buscar en MEDLINE/PubMed. Cada algoritmo de búsqueda fue complementado con palabras clave relevantes que no fueron consideradas como un término MeSH, DeCS o conceptos suplementarios. Los términos fueron: si corresponde, truncados, combinados o ambos con operadores booleanos.

La búsqueda se restringió a publicaciones en español e inglés sin delimitación geográfica. El período de búsqueda para las bases de datos fue del 01 de enero de 2009 al 01 de diciembre de 2018, considerando la fecha de aprobación de la fexofenadina por la FDA en enero de 2011 y al menos 3 años previos para la etapa de pruebas.

Los criterios de inclusión para los estudios fueron los siguientes: a) estudios basados en ensayos clínicos aleatorizados, registros clínicos o administrativos sobre RA; b) estudios observacionales: cohortes, estudios transversales, casos y controles, y casos cruzados; c) resumen disponible para su revisión; 
d) realizado en cualquier área geográfica, y e) entre el 01 de enero de 2009 y el 01 de diciembre de 2018.

Los criterios de exclusión para los estudios fueron: a) estudios publicados a partir de encuestas; b) resúmenes de conferencias, editoriales o comentarios al editor; c) artículos que sólo proporcionan información descriptiva de registros clínicos o administrativos, y d) no presentan desenlaces médicos, como monografías o artículos de revisión.

El equipo de trabajo estuvo integrado por cinco investigadores, y los resúmenes y los textos completos de los estudios identificados fueron revisa-

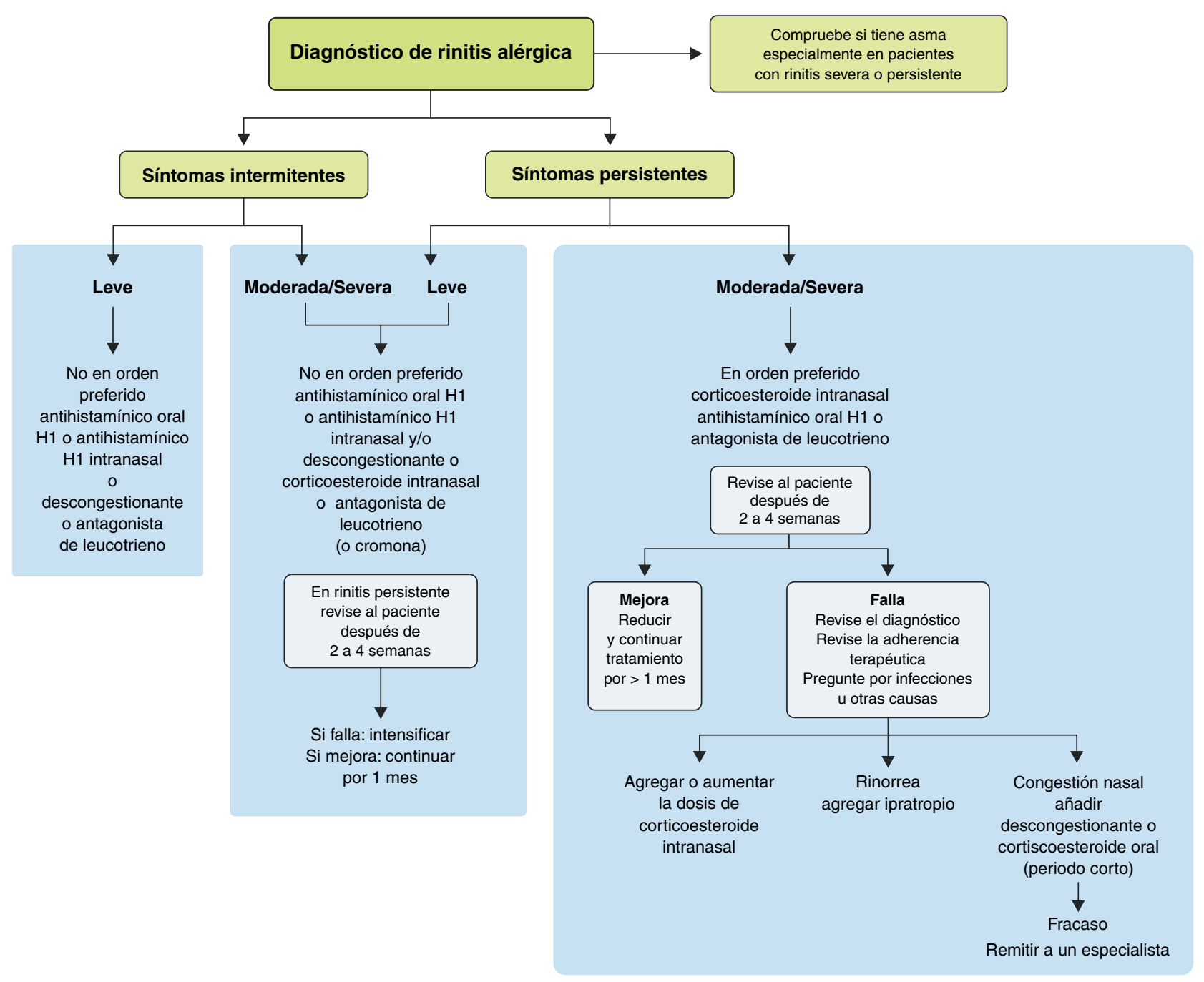

Puede ser apropiado evitar alérgenos e irritantes

\section{Figura 1}

Algoritmo de tratamiento para pacientes con rinitis alérgica Fuente: Bousquet J, et al., 2008. ${ }^{9}$ 
dos en equipos de dos investigadores de forma independiente.

\section{Resultados}

Se seleccionaron 13 artículos con base en el diseño de estudio. Los estudios se llevaron a cabo en adolescentes y adultos; la media de edad de los pacientes fue de 32 años, con un rango de edad desde 15 hasta 85 años. El tiempo promedio de duración de los estudios incluidos fue de 5.1 meses, mientras que el tiempo de duración de las intervenciones farmacológicas presentó una mediana de 2.6 meses (Tabla 1).

Los antihistamínicos orales de segunda generación están recomendados como tratamiento farmacológico de primera línea en los pacientes con rinitis alérgica. ${ }^{13,14}$ Se prefieren por no tener efecto de sedación y reducen especialmente la rinorrea, el prurito y los estornudos; uno de los más usados y estudiados es la fexofenadina. Mösges y colaboradores mencionan la eficacia de la fexofenadina como monoterapia para síntomas nasales (estornudos, prurito, rinorrea, congestión y obstrucción nasal) y síntomas oculares (conjuntivitis). ${ }^{15}$

Por otra parte, los antagonistas de leucotrienos están recomendados también como monoterapia o en combinación con antihistamínicos orales para el manejo de la rinitis alérgica. $9,16-18$

Se han desarrollado combinaciones de antihistamínicos orales con antagonistas de leucotrienos (como fexofenadina, loratadina y levocetirizina, con montelukast), cuyo objetivo es bloquear, además de la liberación de histamina, la producción de cisteinil leucotrienos, los cuales están involucrados en el aumento de la permeabilidad vascular y de la resistencia de la vía aérea. El uso de una terapia combinada tiene la ventaja del doble efecto farmacológico, así como una mejor adherencia al tratamiento al ser administrado de forma conjunta. ${ }^{15,17,19-22}$

La adición de montelukast al tratamiento con antihistamínicos $\mathrm{H} 1$ ha sido evaluada en múltiples ensayos clínicos; a continuación, se describen algunos de ellos.

Ciebiada y colaboradores ${ }^{23}$ realizaron un estudio aleatorizado, doble-ciego, controlado con placebo, cruzado, de dos brazos durante 32 semanas en 40 pacientes con rinitis alérgica persistente, en el que se comparó la monoterapia de montelukast, desloratadina y levocetirizina vs. la combinación de montelukast con los antihistamínicos, y vs. placebo. Demostraron que montelukast en combinación con antihistamínicos tuvo ventajas adicionales en comparación con la monoterapia.

Meltzer y colaboradores, ${ }^{24}$ en un estudio controlado y aleatorizado (ECA) con 460 pacientes con rinitis alérgica estacional (primavera), observaron que la combinación de loratadina con montelukast durante 2 semanas mejoró significativamente los síntomas nasales, oculares y nocturnos, así como las evaluaciones globales y la calidad de vida, en comparación con placebo. La combinación fue bien tolerada.

Gupta y colaboradores ${ }^{25}$ realizaron un ECA en el que compararon montelukast más levocetirizina vs. levocetirizina en monoterapia durante 6 semanas en 102 pacientes con rinitis alérgica. El cambio en las puntuaciones totales de los síntomas nasales diurnos, los síntomas compuestos y los síntomas nasales nocturnos fue significativamente mayor con la combinación vs. la monoterapia.

La combinación de montelukast - fexofenadina también ha sido evaluada en diversos estudios clínicos que se describen a continuación:

Cingi $\mathrm{C}$ y colaboradores ${ }^{26}$ realizaron un estudio multicéntrico, prospectivo, aleatorizado, comparado con placebo, de grupos paralelos, en el que compararon la monoterapia de fexofenadina $(120 \mathrm{mg} /$ día) vs. la combinación de fexofenadina $(120 \mathrm{mg} /$ día) - montelukast (10 mg/día) y vs. la combinación de fexofenadina (120 mg/día) más placebo por 21 días en 275 pacientes con rinitis alérgica estacional. Los puntos finales del estudio fueron divididos en objetivos: hallazgos en la exploración física y rimanometría anterior; y subjetivos: diario de pacientes para síntomas de rinitis alérgica (bloqueo nasal, prurito nasal, estornudos, rinorrea), evaluación de eficacia y satisfacción con el tratamiento a través de una escala visual análoga (EVA). La terapia combinada tuvo efectos estadísticamente significativos vs. monoterapia con fexofenadina en los objetivos evaluados mediante exploración física: disminución de la congestión nasal $(p<0.001)$, edema de los cornetes $(p<0.045)$. Con respecto al reporte de síntomas en el diario de los pacientes, así como la evaluación de la eficacia del tratamiento, el grupo con la combinación obtuvo mejores resultados: congestión nasal $(p<0.003)$, prurito nasal $(p<0.009)$, estornudos $(p<0.004)$, rinorrea $(p<0.001)$; evaluación de la eficacia comparada con los demás grupos de tratamiento $(p<0.037)$. Los autores concluyeron que la combinación de fexofenadina - montelukast es más eficaz que la monoterapia con 


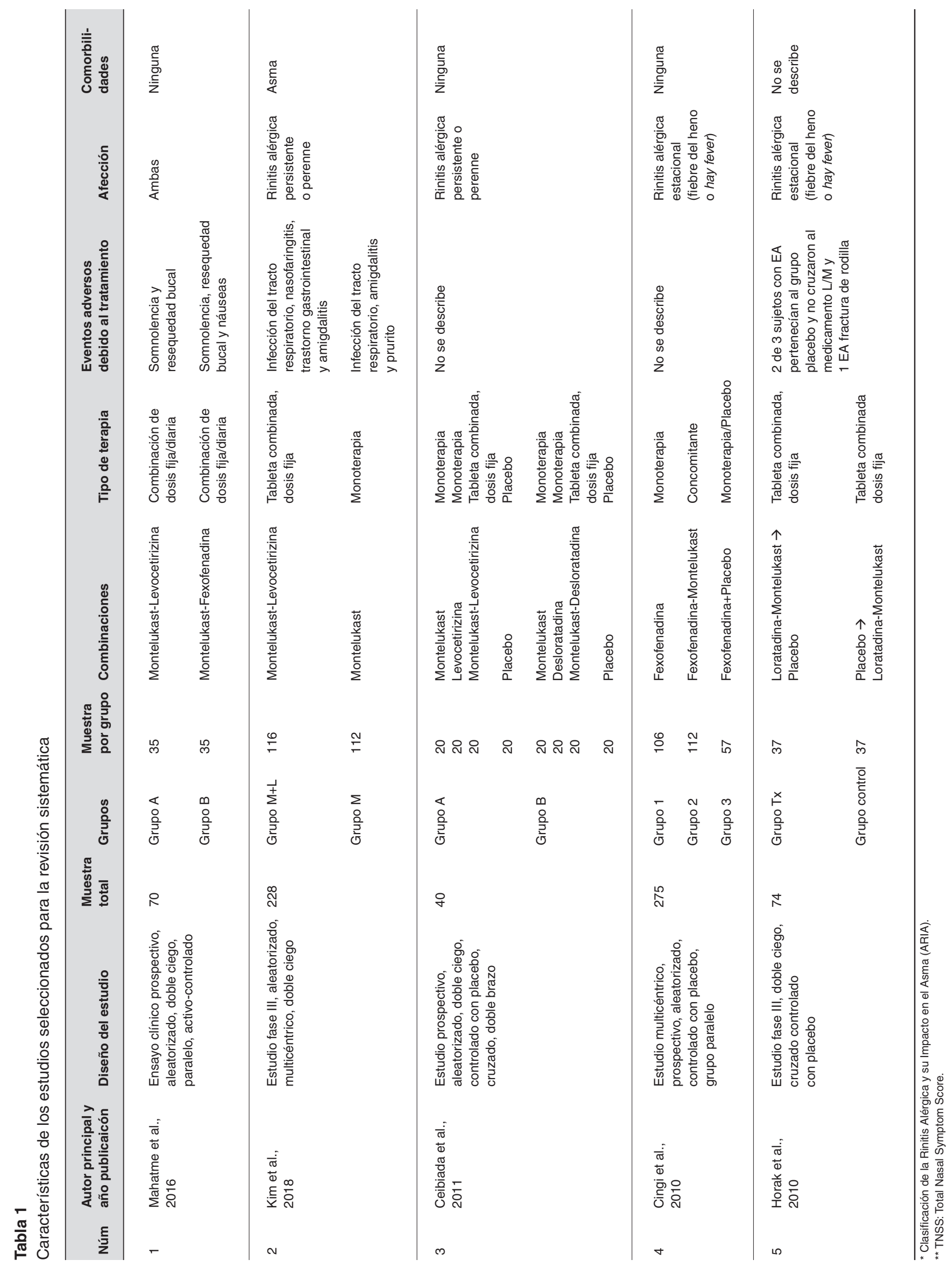




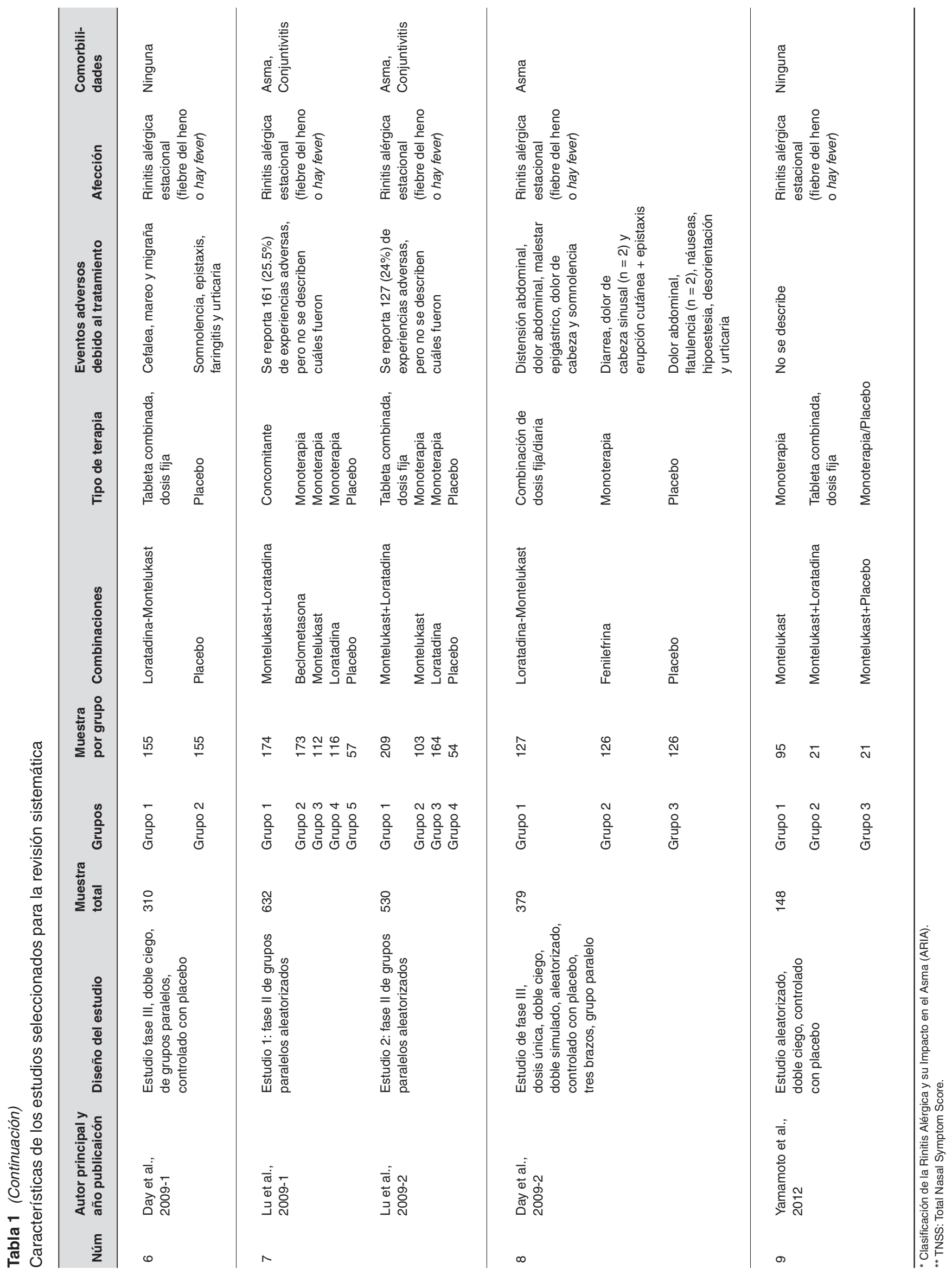




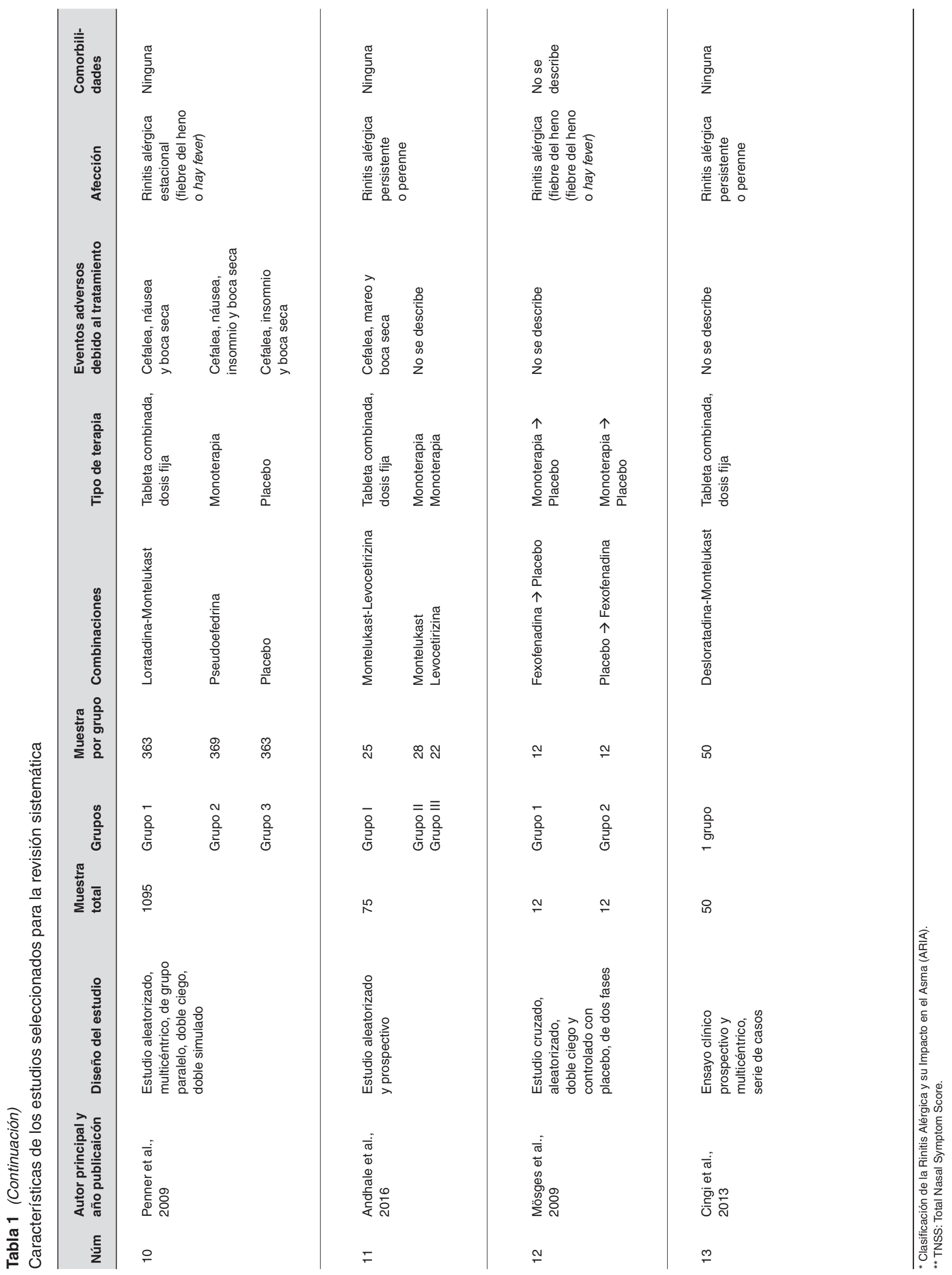


fexofenadina en el control de los síntomas de la rinitis alérgica, principalmente en la congestión nasal.

Naik M y colaboradores ${ }^{27}$ llevaron a cabo un estudio post-marketing, observacional, en la India, en el que evaluaron la eficacia y seguridad de fexofenadina (120 mg) - montelukast (10 mg) en combinación fija (una vez al día) durante 14 días, en 809 pacientes con rinitis alérgica. El objetivo primario de evaluación fue el cambio en la puntuación total de síntomas (suma de la puntuación total de síntomas nasales más puntuación total de síntomas oculares). Los objetivos secundarios fueron la puntuación total de síntomas nasales (congestión nasal, rinorrea, prurito nasal y estornudos) y la puntuación total de síntomas oculares (prurito/ardor ocular, lagrimeo y enrojecimiento) al final del estudio, comparado con el basal y la evaluación global del médico y del paciente de eficacia y tolerabilidad.

La combinación fija de fexofenadina - montelukast fue significativamente eficaz en la reducción de la suma de la puntuación total de síntomas, puntuación total de síntomas nasales (TNSS, por sus siglas en inglés), y puntuación total de síntomas oculares $(p<0.0001$ para todos los parámetros). El estudio demostró la eficacia de la combinación fija de fexofenadina - montelukast en la reducción de la congestión nasal. La evaluación global de eficacia, tanto para el investigador como para el paciente, fue "de excelente a buena" en $>95 \%$ de los pacientes. La mayoría de la población del estudio reportó "buena" tolerabilidad con la combinación fija.

Los resultados de este estudio demuestran que la combinación fija de fexofenadina - montelukast es una opción de tratamiento efectiva, con adecuado perfil de tolerabilidad para el tratamiento de la rinitis alérgica.

Mahatme MS y colaboradores ${ }^{28}$ realizaron un estudio prospectivo, aleatorizado, doble ciego, de grupos paralelos, con controlador activo, en el que se comparó la eficacia, seguridad y costo-efectividad de la combinación fija de montelukast-levocetirizina vs. la combinación fija de montelukast - fexofenadina durante 4 semanas en 70 pacientes con rinitis alérgica. Los resultados fueron medidos en términos de eficacia, y el parámetro principal de la evaluación fue la puntuación total de síntomas nasales. La seguridad fue evaluada a través del monitoreo y seguimiento del reporte de eventos adversos. Para el análisis de costo-efectividad fueron considerados únicamente los costos directos. La puntuación total de síntomas nasales con respecto al valor basal disminuyó en ambos grupos de tratamiento; sin embargo, el grupo con montelukast - fexofenadina tuvo una mayor reducción estadísticamente significativa $(p<0.0033)$ (Tabla 2). No se reportaron eventos adversos serios, y no hubo diferencias estadísticamente significativas en la incidencia de eventos adversos entre los grupos. La relación costo-efectividad fue menor en el grupo con la combinación de montelukast-levocetirizina. Se concluyó que la combinación fija de montelukast - fexofenadina es más eficaz en el control de los síntomas de la rinitis alérgica, aunque la combinación de montelukast-levocetirizina resultó ser más costo-efectiva.

Ciebiada $\mathrm{M}$ y colaboradores ${ }^{29}$ realizaron un estudio prospectivo, cruzado, en el que compararon

Tabla 2

Comparación de la puntuación total de síntomas nasales a las 4 semanas en pacientes con rinitis alérgica

\begin{tabular}{|c|c|c|c|}
\hline \multirow[b]{2}{*}{ Parámetro } & \multicolumn{2}{|c|}{ Cambio de la medición basal a las 4 semanas, media (DE) } & \multirow[b]{2}{*}{ p } \\
\hline & Grupo A $(n=33)$ & Grupo B $(n=32)$ & \\
\hline TNSS & $8.0(2)$ & $9.46(1.41)$ & 0.0033 \\
\hline IC & $7.29-8.70$ & $8.951-9.987$ & \\
\hline
\end{tabular}

$p<0.05$ comparado con Grupo A, prueba de la suma de rangos de Mann-Whitney.

Grupo A: montelukast $10 \mathrm{mg}$ más levocetirizina $5 \mathrm{mg}$.

Grupo B: montelukast $10 \mathrm{mg}$ más fexofenadina $120 \mathrm{mg}$.

TNSS: puntuación total de síntomas nasales.

DE: desviación estándar, IC: intervalo de confianza.

Fuente: Mahatme MS, et al., $2016^{28}$. 
fexofenadina (120 mg/día) más fluticasona $(200 \mu \mathrm{g} /$ día) vs. fexofenadina (120 mg/día) - montelukast (10 mg/día) en dos periodos de 6 semanas cada uno, en 40 pacientes con rinitis alérgica persistente y asma de diagnóstico reciente. Los objetivos primarios de eficacia consistieron en la concentración sérica de la molécula de adhesión intracelular tipo-1 (sICAM-1), puntuación total de síntomas nasales (TNSS), puntuación de síntomas de asma y puntuación de rinoscopia anterior. Los objetivos secundarios, uso de medicamentos concomitantes y eventos adversos.

Dentro de los resultados, se encontró que ambos grupos de tratamiento fueron eficaces en la mejoría y reducción de todos los puntos evaluados en los objetivos primarios, sin diferencias estadísticamente significativas entre ellos (fexofenadina más fluticasona: basal, $243.4 \pm 20.7$; después del tratamiento, $221 \pm 20.4$; fexofenadina más montelukast: basal, $240.9 \pm 19.8$; después del tratamiento, $218.3 \pm 17.6)$. Por otro lado, no se reportaron eventos adversos en ninguno de los dos grupos, y no hubo diferencias entre los grupos respecto al uso de medicamentos de rescate. Los autores concluyeron que la adición de montelukast al tratamiento con fexofenadina produjo la misma mejoría en todos los parámetros evaluados vs. la adición de fluticasona a fexofenadina en este grupo de pacientes con rinitis alérgica persistente y asma de recién diagnóstico.

Recientemente se llevó a cabo una revisión sistemática en la que se incluyeron las monoterapias de fexofenadina y montelukast, así como la combinación de montelukast - fexofenadina, y montelukast con otros antihistamínicos de segunda generación. Los resultados corroboran que estos fármacos en monoterapia y en combinación son eficaces y seguros en pacientes con rinitis alérgica. ${ }^{30}$

\section{Conclusiones}

La principal contribución de este trabajo es que reúne evidencias de la eficacia y seguridad del uso de terapias combinadas fijas de antihistamínico con antagonistas de leucotrienos, lo cual favorece también el apego terapéutico, considerando la evidencia disponible que plantea que las combinaciones fijas mejoran el apego al tratamiento en comparación con las terapias libres y, a largo plazo, ayudan a alcanzar el objetivo propuesto. ${ }^{31} \mathrm{Al}$ tratarse de una enfermedad respiratoria crónica es fundamental identificar cuál es la mejor combinación de fármacos para el manejo y control que permita mejorar la calidad de vida de los pacientes que la padecen, así como una menor incidencia de eventos adversos.

- A partir de la evidencia científica disponible, se concluye que las monoterapias de fexofenadina y montelukast son eficaces y seguras en el control de los síntomas de pacientes con rinitis alérgica.

- Las combinaciones de antihistamínicos de segunda generación (fexofenadina, loratadina y levocetirizina) con antagonistas de leucotrienos (montelukast) son eficaces y seguras en el control de la rinitis alérgica, además de ser una alternativa que ofrece la ventaja de una mejor adherencia terapéutica para los pacientes con este padecimiento.

\section{Referencias}

1. Organización Mundial de la Salud (OMS). OMS - Enfermedades respiratorias crónicas. WHO, 2016 [Internet]. Disponible en: https://www.who.int/respiratory/about_topic/es/. Consultado el 05/12/2019.

2. World Health Organization (WHO). Action plan of the Global Alliance against Chronic Respiratory Diseases. Action Plan 2008-2013. Italy: World Health Organization, (WHO); 2008. p. 34.

3 Dykewicz MS, Hamilos DL. Rhinitis and sinusitis. J Allergy Clin Immunol. 2010;125(2):S103-15.

4. Akdis CA, Hellings PW, Agache I, eds.; European Academy of Allergy and Clinical Immunology (EAACl). Global atlas of allergic rhinitis and chronic rhinosinusitis. Zurich: EAACl; 2015.

5. U.S. National Library of Medicine (NLM). Enciclopedia médica. Rinitis alérgica. U.S. National Library of Medicine (NLM); 2018.

6. Wheatley LM, Togias A. Allergic rhinitis. N Engl J Med. 2015;372(5):456-63. doi: 10.1056/NEJMcp1412282.

7. Brozek JL, Bousquet J, Agache I, Agarwal A, Bachert C, BosnicAnticevich S, et al. Allergic Rhinitis and its Impact on Asthma (ARIA) guideline - 2016 revision. J Allergy Clin Immunol. 2017;140(4):950-8.

8. van Cauwenberge P, Bachert C, Passalacqua G, Bousquet J, Canonica GW, Durham SR, et al. Consensus statement on the treatment of allergic rhinitis. European Academy of Allergology and Clinical Immunology. Allergy. 2000;55(2):116-34.

9. Bousquet J, Khaltaev N, Cruz AA, Denburg J, Fokkens WJ, Togias A, et al. Allergic Rhinitis and its Impact on Asthma (ARIA) 2008. Allergy. 2008;63(Suppl. 86):8-160. doi:10.1111/j.13989995.2007.01620.x

10. Meltzer EO. Quality of life in adults and children with allergic rhinitis. J Allergy Clin Immunol. 2001;108(1 Suppl):S45-53.

11. Camelo-Nunes IC, Solé D. Rinite alérgica: indicadores de qualidade de vida. J Bras Pneumol. 2010;36(1):12-33.

12. Xiao J, Wu WX, Ye YY, Lin WJ, Wang L. A network meta-analysis of randomized controlled trials focusing on different allergic rhinitis medications. Am J Ther. 2016;23(6):e1568-e1578.

13. Small $P$, Keith PK, Kim H. Allergic rhinitis. Allergy, Asthma Clin Immunol. 2018;14(2):51. doi:10.1186/s13223-018-0280-7.

14. México, Instituto Mexicano del Seguro Social. Diagnóstico y Tratamiento de Rinitis Alérgica. Guía de Evidencias y Recomendaciones: Guía de Práctica Clínica. [Internet] México, IMSS;2017. Disponible en: http://www.imss.gob.mx/profesionalessalud/gpc. 
15. Mösges R, Lehmacher W, Pasch N, Vent J. Assessment of the antiobstructive effect of fexofenadine on nasal allergy challenge in patients with seasonal allergic rhinitis. Asian Pacific J Allergy Immunol. 2009;27(4):1-10.

16. Escuela Andaluza de Salud Pública. Nuevos tratamientos del asma: antagonistas de los leucotrienos. Bol Ter Andal. 1999;15(6):21-2.

17. Walekar A, Chodankar D, Naqvi M, Trivedi C. Assessment of Bioequivalence of Fexofenadine and Montelukast Fixed Dose Combination Tablet Versus Separate Formulations of the Individual Components at the Same Dose Levels. Indian J Pharm Sci. 2016;78(5):651-6.

18. Baños ZM, Somonte ZDE, Morales PV. Leucotrienos y antileucotrienos en medicina basada en la evidencia. Rev Mex Patol Clin Med Lab. 2015;62(3):157-62.

19. Takahashi G, Matsuzaki Z, Okamoto A, Ito E, Matsuoka T, Nakayama T, et al. A randomized control trail of stepwise treatment with fluticasone propionate nasal spray and fexofenadine hydrochloride tablet for seasonal allergic rhinitis. Allergol Int. 2012;61(1):155-62.

20. Horak F, Zieglmayer P, Zieglmayer R, Lemell P. Onset of action of loratadine/montelukast in seasonal allergic rhinitis patients exposed to grass pollen. Arzneimittelforschung. 2010;60(5):249-55.

21. Yamamoto H, Yamada T, Sakashita M, Kubo S, Susuki D, Tokunaga $\mathrm{T}$, et al. Efficacy of prophylactic treatment with montelukast and montelukast plus add-on loratadine for seasonal allergic rhinitis. Allergy Asthma Proc. 2012;(c):17-22.

22. Kim MK, Lee SY, Park HS, Yoon HJ, Kim SH, Cho YJ, et al. A Randomized, Multicenter, Double-blind, Phase III Study to Evaluate the Efficacy on Allergic Rhinitis and Safety of a Combination Therapy of Montelukast and Levocetirizine in Patients With Asthma and Allergic Rhinitis. Clin Ther. 2018;40(7):1096-107.

23. Ciebiada M, Gorska-Ciebiada M, Barylski M, Kmiecik T, Gorski P. Use of montelukast alone or in combination with desloratadine or levocetirizine in patients with persistent allergic rhinitis. Am J Rhinol Allergy. 2011;25(1):1-6.

24. Meltzer EO, Malmstrom K, Lu S, Prenner BM, Wei LX, Weinstein $\mathrm{SF}$, et al. Concomitant montelukast and loratadine as treatment for seasonal allergic rhinitis: a randomized, placebo-controlled clinical trial. J Allergy Clin Immunol. 2000;105(5):917-22.

25. Gupta V, Matreja PS. Efficacy of montelukast and levocetirizine as treatment for allergic rhinitis. J Aller Ther. 2010;1:103. doi: 10.4172/2155-6121.1000103.
26. Cingi C, Gunhan K, Gage-White L, Unlu H. Efficacy of leukotriene antagonists as concomitant stherapy in allergic rhinitis. Laryngoscope. 2010;120(9):1718-23. doi:10.1002/lary.20941.

27. Naik M, Nayak A, Khandeparkar P, Mukaddam Q. Efficacy and safety of montelukast plus fexofenadine fixed dose combination in allergic rhinitis: Results of post-marketing study in India. Indian Medical Gazette. 2013;8:314-8.

28. Mahatme MS, Dakhale GN, Tadke K, Hiware SK, Dudhgaonkar S, Wankhede S. Comparison of efficacy, safety, and cost-effectiveness of montelukast-levocetirizine and montelukast-fexofenadine in patients of allergic rhinitis: A randomized, double-blind clinical trial. Indian J Pharmacol. 2016;48(6):649-53. doi:10.4103/02537613.194854.

29. Ciebiada M, Gorska-Ciebiada M, Gorski P. Fexofenadine with either montelukast or a low-dose inhaled corticosteroid (fluticasone) in the treatments of patients with persistent allergic rhinitis and newly diagnosed asthma. Arch Med Sci. 2009;5:564-9.

30. González-González E, García-Morales C, Molina-Vélez D, et al. Protocolo de revisión sistemática de literatura sobre la eficacia y seguridad de las monoterapias de fexofenadina y montelukast, y la terapia de montelukast en combinación con fexofenadina $u$ otros antihistamínicos de segunda generación para el tratamiento. 2019. [Internet]. Disponible en: https://osf.io/mfzdp/. Consultado el 24/02/2020.

31. Escobar C, Barrios V. Combined therapy in the treatment of hypertension. Fundam Clin Pharmacol. 2010;24:3-8.4.

\section{Conflicto de Intereses}

Ninguno de los autores de este proyecto presenta conflicto de intereses para participar en el estudio, a excepción de las autoras Vanessa Cohen-Muñoz y Gabriela Sánchez-Casado, que son empleadas de Sanofi Aventis de México, S.A. de C.V.

Autor correspondiente:

Leonel González-González

E-mail: leonel.gonzalez@insp.mx 